\title{
van der Waals Interaction Activated Strong Electronic Coupling at the Interface between Chloro Boron-Subphthalocyanine and $\mathrm{Cu}(111)$
}

\author{
Shashank S. Harivyasi, ${ }^{\dagger} \odot$ Oliver T. Hofmann, ${ }^{\dagger}{ }^{\oplus}$ Nahid Ilyas, ${ }^{\ddagger}$ Oliver L. A. Monti, ${ }^{\ddagger}, \odot$ \\ and Egbert Zojer* ${ }^{*} \odot$
}

\author{
${ }^{\dagger}$ Institute of Solid State Physics, NAWI Graz, Graz University of Technology, Petersgasse 16, 8010 Graz, Austria \\ ${ }^{*}$ Department of Chemistry and Biochemistry, University of Arizona, 1306 E. University Blvd., Tucson, Arizona 85721, United States \\ ${ }^{\S}$ Department of Physics, University of Arizona, 1118 E 4th Street, Tucson, Arizona 85721, United States
}

\section{Supporting Information}

\begin{abstract}
In this article, we investigate the interface between shuttlecock-shaped chloro boron-subphthalocyanine molecules and the $\mathrm{Cu}(111)$ surface. We highlight how molecular planarization induced by van der Waals forces can fundamentally alter the interface properties and how it can enable a particularly strong hybridization between molecular and metal states. In our simulations, we start from a situation in which we disregard van der Waals forces and then introduce them gradually by rescaling the interaction parameter, thereby "pulling" the molecule toward the surface. This reveals two adsorption regimes with significantly different adsorption distances, molecular conformations, and adsorbate-induced changes of the work function. Notably, the above-mentioned massive hybridization of electronic states, also observed in photoelectron spectroscopy, is obtained solely for one of the regimes. We show that this regime is accessible only as a consequence of the planarization of the molecular backbone resulting from the van der Waals attraction between the molecule and the surface. The results of this study indicate that for certain metal-molecule combinations unusually strong interfacial electronic interactions can be triggered by van der Waals forces creating a situation that differs from the usually described cases of physisorptive and chemisorptive interactions.
\end{abstract}

\section{INTRODUCTION}

In organic (opto)electronic devices, molecules are often in direct contact with metal electrodes. This makes a comprehensive understanding of metal-organic interfaces a prerequisite for improving device performance. A key factor that dominates the properties of such interfaces is the strength of the interaction between the metal and the molecules. Together with the electronic structure of these interfaces, the interactions have been explained by invoking a number of phenomena, such as exchange interaction, dipole interaction, charge transfer, and covalent bonding. ${ }^{1-10}$

The present study focuses on understanding peculiarities of the interfacial interactions and the electronic structure that may arise from adsorption-induced geometry changes in certain nonplanar molecules. In particular, we present a detailed computational study of the adsorption of chloro boronsubphthalocyanine (ClB-SubPc) on $\mathrm{Cu}(111)$ and corroborate it with an assessment of the interfacial electronic structure via photoelectron spectroscopy. The system serves as a prototypical example for the hitherto rarely discussed ${ }^{11}$ scenario in which the electronic structure of an interface is fundamentally altered by changes of molecular conformation that are, in turn, enabled by the van der Waals (vdW) forces between the molecule and the substrate.

Beyond the fundamental character of the present study, it is also motivated by the increasing technological relevance of subphthalocyanines (SubPcs), which calls for an improved understanding of their properties. SubPcs are optically active dyes that exhibit fluorescence and a high second-order linear response. $^{12,13}$ They have been used in organic photovoltaic cells, ${ }^{14}$ in light-harvesting systems, ${ }^{15}$ and for blending-driven band gap engineering. ${ }^{16}$ Consequently, film formation of the archetypical subphthalocyanine, $\mathrm{ClB}-\mathrm{SubPc}$, on different metal surfaces has been the focus of numerous studies, ${ }^{17-22}$ all revealing feature-rich thin films. The latter is, at least in part, a consequence of the molecule's peculiar geometry: A ClB-SubPc molecule has a threefold rotational symmetry, combined with a conical "shuttlecock-shaped" nonplanar geometry (Figure 1).

Consequently, depending on the molecular orientation with respect to the substrate upon adsorption, multiple plausible adsorption situations can be envisaged. ${ }^{22}$ This results, for

Received: April 18, 2018

Revised: May 18, 2018

Published: May 24, 2018 


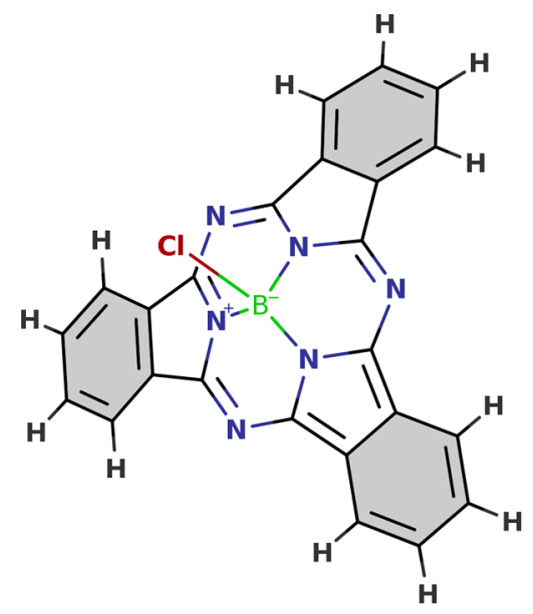

Figure 1. Chemical structure of chloro boron-subphthalocyanine (ClB-SubPc). Upon adsorption, the 18 carbon atoms of the three benzene subunits of the molecule (shaded gray here) are found closest to the $\mathrm{Cu}$ substrate. They are collectively referred to as $\mathrm{C}-18$ in this article.

example, in charge transfer from the surface to the chlorine atom on $\mathrm{Ag}(111),{ }^{18}$ in the formation of bilayer nanocrystals on $\mathrm{Cu}(111),{ }^{20}$ or in surface-catalyzed dechlorination of (a fraction of) the molecules on $\mathrm{Cu}(111) .^{22}$ In the last case, one observes the formation of chemically different ClB-SubPc domains consisting of either dechlorinated or intact molecules. ${ }^{22}$ Here, we focus on molecules that remain intact while adsorbing on $\mathrm{Cu}(111)$, while the most relevant data for dechlorinated molecules are compiled in the Supporting Information (SI). The case of intact molecules is particularly insightful for understanding the peculiarities that can arise from the interaction between a nonplanar $\pi$-electron system and a coinage metal substrate: By tuning the magnitude of van der Waals forces when simulating the ClB-SubPc/ $\mathrm{Cu}(111)$ interface, we are able to show how the van der Waals-induced planarization of (significant parts of) the molecular backbone upon adsorption gives rise to two distinct adsorption regimes. Moreover, the planarization enables a particularly strong electronic coupling between substrate and adsorbate. This, in turn, fundamentally changes interface properties like the adsorbate-induced work function modification, the local density of states, and the interfacial charge rearrangement.

\section{METHODOLOGY}

2.1. Computational. For our simulations, we relied on density functional theory (DFT) as implemented in the Fritz Haber Institute $a b$ initio molecular simulations (FHI-aims) package, ${ }^{23}$ employing the default "tight" settings. We used the Perdew-Burke-Ernzerhof (PBE) functional ${ }^{24}$ complemented by a surface-screening-corrected van der Waals energy term, ${ }^{25}$ a

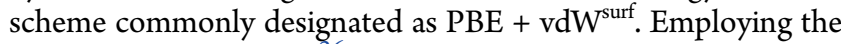
repeated-slab approach, ${ }^{26}$ we studied the adsorption process at a moderate packing density ${ }^{22}$ with just one molecule in every 7 $\times 4 \sqrt{3}$ orthogonal surface unit cell. This represents the smallest orthogonal cell in which the energy contribution from intermolecular interactions has decayed to less than $0.1 \mathrm{eV}$. For simulating the isolated molecule in the gas phase, we also used the FHI-aims package and employed open boundary conditions with equivalent computational settings (the resulting coordinates are provided in the SI).
The $\mathrm{Cu}(111)$ surface was represented by a slab with a thickness of six atomic layers, of which only the top two were allowed to relax in the geometry optimizations. In the direction perpendicular to the surface, a vacuum gap of at least $25 \AA$ was introduced together with a self-consistently determined discontinuity in the electrostatic energy to decouple periodic replicas of the slab. ${ }^{27}$ The convergence criterion for the selfconsistent field cycles was set to $10^{-6} \mathrm{eV}$ for the total energy, and the geometry was relaxed until the maximum residual force component per atom was less than $0.01 \mathrm{eV} / \AA ̊$. The states were broadened using a Gaussian function, and also a Gaussian occupation scheme ${ }^{28}$ was employed. For both, $\sigma$ was set to 0.2 eV. A $4 \times 4 \times 1 \Gamma$-centered $k$-point grid was used for all periodic calculations, and van der Waals corrections were not included for $\mathrm{Cu}-\mathrm{Cu}$ atom pairs in calculations containing the $\mathrm{Cu}$ slab. The lattice constant for $\mathrm{Cu}$, obtained with the above settings is $3.637 \AA$.

2.2. Experimental Section. ClB-SubPc was purchased from Sigma-Aldrich and was purified by repeated gradient sublimation in a custom-built furnace. It was degassed overnight in a home-built Knudsen cell under ultrahigh vacuum conditions and at temperatures slightly below the sublimation point. A polished $\mathrm{Cu}(111)$ crystal of $99.999 \%$ purity (Princeton Scientific) was cleaned by repeated cycles of $\mathrm{Ar}^{+}$sputtering and annealing, whereas highly oriented pyrolytic graphite (HOPG) was freshly cleaved and annealed at $450{ }^{\circ} \mathrm{C}$ in vacuum. Molecular deposition occurred at room temperature on both substrates, at a rate of $0.1 \AA / \mathrm{min}$. Photoelectron spectroscopy was carried out using a $\mathrm{He}$ (I) UV source (SPECS UVS 10/35) incident at a $30^{\circ}$ angle in a VG EscaLab MK II photoelectron spectrometer. Spectra were acquired with an energy resolution of $89(8) \mathrm{meV}$.

\section{RESULTS AND DISCUSSION}

3.1. Planarization and Hybridization of CIB-SubPc upon Adsorption on $\mathrm{Cu}(111)$. The shuttlecock-shaped geometry of the ClB-SubPc molecule (Figure 2a) is lost once it adsorbs on $\mathrm{Cu}(111)$. The molecule then resembles a planarized disk with only a trigonal pyramidal feature (composed of chlorine, boron, and three pyrrolic nitrogen atoms) preserved in its center (Figure 2b). The 18 carbon atoms closest to the substrate (collectively termed as C-18; see shaded rings in Figure 1) end up lying approximately in the same plane, with the standard deviation in their $z$-coordinates $\sigma_{\mathrm{C}-18}=\sqrt{\frac{1}{18} \sum_{i}^{18}\left(\mathbf{z}_{i}-\overline{\mathbf{z}}_{i}\right)^{2}}$ as low as $0.03 \AA$. As a more accessible quantification, in the following we define the planarity, $\Pi$, of any given $\mathrm{ClB}-\mathrm{SubPc}$ conformation via the standard deviation in the heights of the C-18 atoms as

$$
\Pi=\frac{\sigma_{\mathrm{C}-18, \text { gas-phase }}-\sigma_{\mathrm{C}-18, \text { given-geometry }}}{\sigma_{\mathrm{C}-18, \text { gas-phase }}} \times 100 \%
$$

This definition implies that the molecule in its gas-phase geometry has a planarity, $\Pi$, of $0 \%$, whereas $\Pi$ is $100 \%$ when all $\mathrm{C}-18$ atoms are in the same plane. For the relaxed ClB-SubPc/ $\mathrm{Cu}(111)$ interface, a planarity of $94 \%$ is obtained for the adsorbed molecule. Concomitantly, the molecule's "shuttlecock angle", the obtuse angle between the central symmetry axis and any of the six peripheral carbon atoms in the benzene subunits, decreases by $13^{\circ}$ (from 116 to $103^{\circ}$, Figure 2a,b).

Putting the planarization of the ClB-SubPc $\pi$-system into the context of similar molecules is difficult. With three instead of 


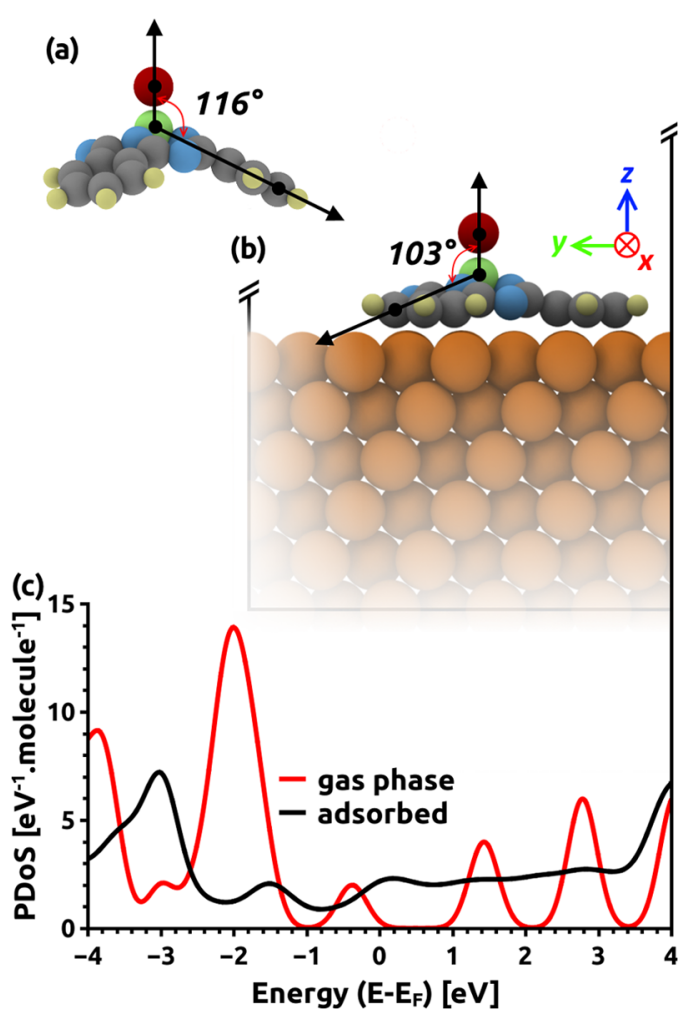

Figure 2. Structure of the ClB-SubPc molecule (a) before and (b) after adsorption on $\mathrm{Cu}(111)$. (c) Molecule-projected density of states (PDoS) of the ClB-SubPc molecule adsorbed on the $\mathrm{Cu}(111)$ substrate (black curve) and in the gas phase (red curve). The DoS of the gas-phase molecule has been Gaussian broadened and shifted such that the vacuum level of the molecule is the same as that of a clean $\mathrm{Cu}$ slab. The Fermi level of that slab is then used as the energy reference for the isolated molecule in the above plot. Panel (b) also indicates the unit cell and the coordinate system used in all calculations employing periodic boundary conditions. The coloring scheme is as follows: chlorine, red; boron, green; nitrogen, blue; carbon, gray; hydrogen, yellow; and copper, brown.

four isoindole units and meso $\mathrm{N}$ atoms, boron-subphthalocyanines are, so far, the only known lower homologues of phthalocyanine $(\mathrm{Pc})$ molecules. Although the adsorption of numerous nonplanar Pcs has been studied extensively, ${ }^{29-40}$ most have comparably smaller shuttlecock angles and thus do not lend themselves to direct comparison. For instance, the nearest comparable study of $\mathrm{SnPc}$ on $\mathrm{Ag}(111)$ also employing dispersion-corrected DFT reports that adsorption results in a change of $5^{\circ}$ (from 105 to $100^{\circ}$ ) in the molecule's shuttlecock angle when it adsorbs in a similar orientation. ${ }^{36}$ On the other extreme, there are spherical- and bowl-shaped molecules like $\mathrm{C}_{60}$ and corannulene $\left(\mathrm{C}_{20} \mathrm{H}_{10}\right)$ that appear not to flatten on $\mathrm{Cu}(111)$, and for which the overall geometry remains largely unchanged upon adsorption. ${ }^{41,42}$

Besides planarization, the other significant consequence of adsorption of ClB-SubPc molecules on $\mathrm{Cu}(111)$ is the change in their electronic structure (Figure 2c). Notably, adsorption leads to a substantial "smearing" of the molecule-projected DoS (PDoS) around the Fermi level of the metal-molecule system. This occurs to the extent that different molecular states are difficult to identify both in the PDoS and in the measured ultraviolet photoelectron (UP) spectrum (see below). The particularly strong smearing of the PDoS is caused by the hybridization of occupied as well as unoccupied molecular states with the d-band and the sp-band of copper. Similar observations have been made on $\mathrm{Cu}$ substrates for other conjugated molecules, which are flat in the gas phase. The relevant interfaces include, for example, pentacene on $\mathrm{Cu}(111),{ }^{7,43} \mathrm{Cu}(100),{ }^{44}$ and $\mathrm{Cu}(110),{ }^{45,46}$ as well as perylenetetracarboxylic dianhydride (PTCDA) on $\mathrm{Cu}(111){ }^{9,47}$

Additionally, the ClB-SubPc monolayer is rendered metallic because the Fermi level lies in a region of significant PDoS. Metallization of the interface due to strong hybridization is a commonly observed feature for planar molecules adsorbed on metal surfaces. ${ }^{7,9,43-50}$ In fact, a previous investigation of ClBSubPc on $\mathrm{Cu}(111)$ using two-photon photoemission spectroscopy also suggested hybridization and charge transfer to the molecular orbitals from metal surface states. ${ }^{21}$

The strong broadening of the PDoS found in the computational results is also present in the experimental data. In Figure 3, we show a comparison of the UP spectra of 0.7

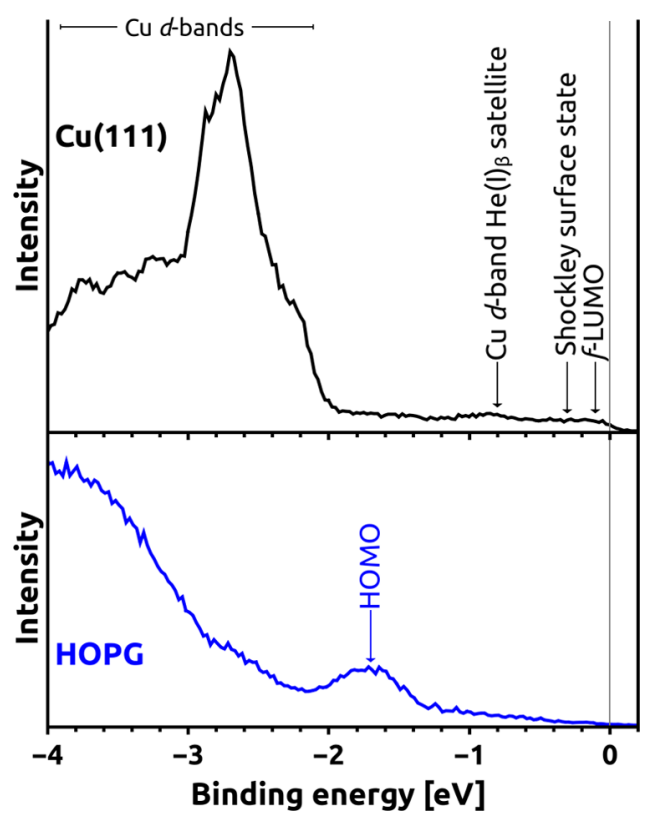

Figure 3. Ultraviolet photoelectron spectra of a ClB-SubPc monolayer on $\mathrm{Cu}(111)$ (top panel, black, strong hybridization) and HOPG (bottom panel, blue, weak hybridization). The binding energy is referenced to the Fermi level.

monolayer equivalents (as defined in ref 22) of ClB-SubPc on both $\mathrm{Cu}(111)$ and HOPG. On HOPG, a distinct feature representing the $\mathrm{ClB}-\mathrm{SubPc}$ highest occupied molecular orbital (HOMO) centered at a binding energy $\left(E_{\mathrm{B}}\right)$ of $-1.7 \mathrm{eV}$ is clearly resolved on top of the rather weak background originating from the HOPG $\pi$-band. This is consistent with a UP spectroscopy report on a $7.5 \mathrm{~nm}$ thick ClB-SubPc film on $\mathrm{Si}^{51}$ Further, peaks are observed at higher binding energies. In contrast, on $\mathrm{Cu}(111)$ only weak and broad features are visible between 0 and $-2 \mathrm{eV}$. These stem primarily from the $\mathrm{Cu} \mathrm{d}$ band $\mathrm{He}(\mathrm{I})_{\beta}$ satellite features at $-0.8 \mathrm{eV}$. Additional contributions from the remainder of the $\mathrm{Cu}(111)$ Shockley surface state $(-0.2$ to $-0.4 \mathrm{eV})$ and from a partially filled lowest unoccupied molecular orbital near the Fermi edge are expected at the positions indicated in Figure 3 following the two-photon photoemission data in ref 21 . Below $-2 \mathrm{eV}$, the $\mathrm{Cu}$ $\mathrm{d}$-bands manifest as the strongest features in the ClB-SubPc/ $\mathrm{Cu}(111) \mathrm{UP}$ spectrum. Their broad structure is indicative of 
scattering in the organic adlayer and interfacial hybridization. There is no clear evidence of the HOMO or other molecular features in the UP spectrum, though a contribution from the $\mathrm{HOMO}$ to the shoulder at $E_{\mathrm{B}}=-2.2 \mathrm{eV}$ or the d-band region cannot be excluded (we do not necessarily expect the HOMO feature on $\mathrm{Cu}(111)$ to be at the same energy as on HOPG). In summary, the distinctly different contributions of ClB-SubPc to the UP spectra when adsorbed on HOPG and $\mathrm{Cu}(111)$ support the notion of a strong smearing of the molecular states at the $\mathrm{ClB}-\mathrm{SubPc} / \mathrm{Cu}(111)$ interface that has been inferred from the simulations.

The above observations raise the question to what extent the particularly strong coupling between substrate and adsorbate and the interaction-induced planarization of most of the molecular backbone are interrelated. To address this issue, as a first step we calculated the adsorption energy $E_{\text {ads }}$ as a function of the adsorption height $h_{\text {ads }}$. We define $E_{\text {ads }}$ as

$$
E_{\mathrm{ads}}=E_{\text {inter }}-\left(E_{\mathrm{Cu}}+E_{\mathrm{mol}}\right)
$$

where $E_{\text {inter }}$ is the energy of the interacting metal-molecule system, $E_{\mathrm{Cu}}$ is the energy of a pristine $\mathrm{Cu}(111)$ slab (again, with only the top two layers relaxed), and $E_{\text {mol }}$ is the energy of a single relaxed ClB-SubPc molecule in the gas phase. In addition, the methodology applied to treat van der Waals interactions allows us to compute separately the contribution of these energies to $E_{\mathrm{ads}}$, which we term as $E_{\mathrm{vdW}}$ (see SI for details).

The adsorption height, $h_{\mathrm{ads}}$, is defined as the difference between the average heights of the C-18 atoms and that of the metal atoms in the topmost layer of the reconstructed substrate. At equilibrium, this height is $2.23 \AA$, which appears low at first glance, but it is comparable to adsorption distances measured for planar $\pi$-conjugated systems in similar situations, such as the $2.34 \AA$ found for pentacene on $\mathrm{Cu}(111) .^{52}$

In practice, to trace $E_{\mathrm{ads}}$ vs $h_{\mathrm{ads}}$, we gradually moved the molecule from its equilibrium position along the z-axis in steps of $0.2 \AA$, fixed the position of the $\mathrm{Cl}$ atom, and then optimized the geometry of the rest of the molecule as well as the top two atomic layers of the $\mathrm{Cu}$ substrate. The resulting curve, shown in Figure 4a, displays a few peculiarities: (i) The PBE contribution to the adsorption energy (green curve) is positive for all distances. This hints at a distinctly repulsive interaction between the ClB-SubPc molecules and the $\mathrm{Cu}(111)$ surface in the absence of long-range van der Waals interactions. In fact, we observe a detachment of the molecule from the surface, when van der Waals (vdW) interactions are turned off and the system geometry is optimized. (ii) The dependence of the adsorption energy on adsorption distance (when including vdW attraction) displays an unusual, essentially linear shape between the equilibrium distance of $2.23 \AA$ and the largest considered distance of $3.35 \AA$ ( $r^{2}=0.996$ for a linear fit plotted in orange). There is also no indication of multiple minima, as are observed for systems displaying physisorbed as well as chemisorbed structures. $^{6,53}$

Further insight can be gained by tracing the planarity of the adsorbed molecules as a function of $h_{\text {ads }}$, as shown in Figure $4 \mathrm{~b}$ (red curve): First, $\Pi$ increases smoothly, as the molecule approaches the surface. However, at ca. $0.2 \AA$ above the equilibrium distance, an increased planarization sets in, as is particularly evident when comparing the actual behavior at short distances with an extrapolation from larger adsorption heights (i.e., for $h_{\mathrm{ads}} \in[2.79,3.35 \AA]$, pink dashed curve). This means that the molecule undergoes a disproportionately large

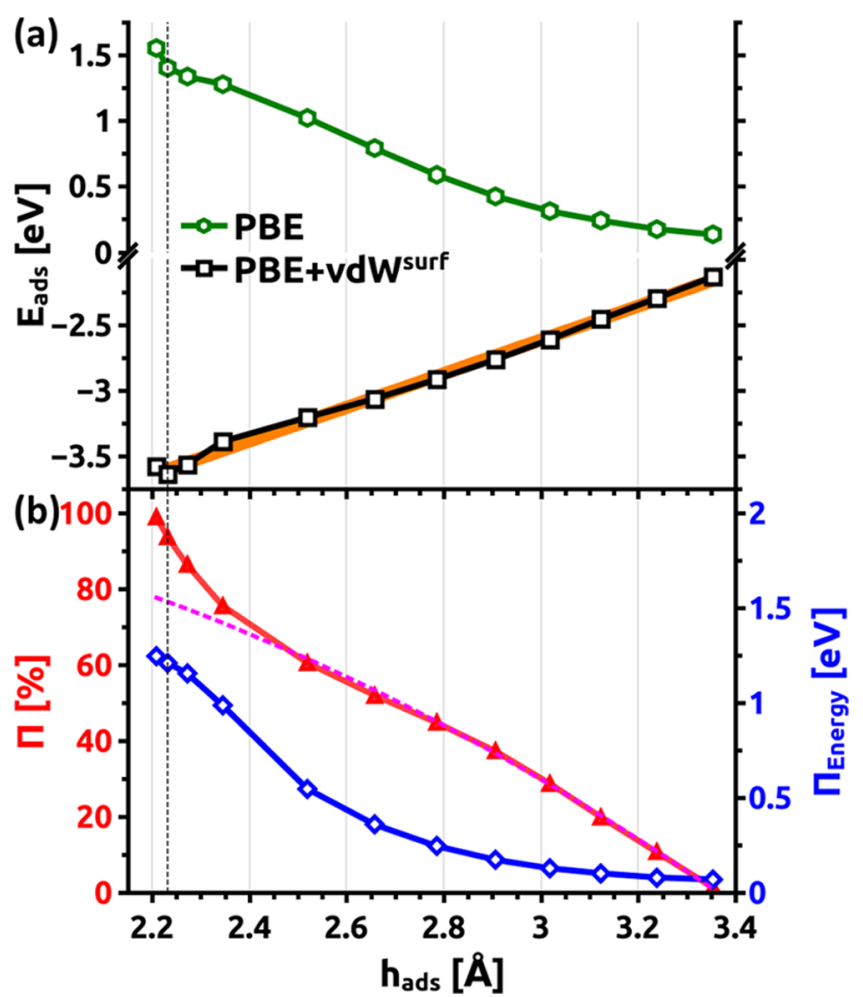

Figure 4. (a) Adsorption energy $\left(E_{\mathrm{ads}}\right)$ per ClB-SubPc molecule as a function of adsorption distance including (black curve) and neglecting (green curve) long-range van der Waals interactions $\left(E_{\mathrm{vdW}}\right)$. The orange line in the background is a linear fit to the $E_{\mathrm{ads}}\left(\mathrm{PBE}+\mathrm{vdW} \mathrm{Wur}^{\text {sur }}\right)$ curve, highlighting the unusual shape of the adsorption potential. (b) Change in the planarity $(\Pi)$ of the molecule as a function of its adsorption height (red triangles). The pink dashed curve indicates the "expected" trend extrapolated from quadratic fitting of the data points with $h_{\text {ads }}>2.7 \AA$. The blue curve and the right axis refer to the energetic cost of planarizing the molecule $\left(\Pi_{\text {energy }}\right)$, i.e., of changing the molecular geometry from that obtained for the gas phase to that found at a specific adsorption distance.

planarization within a very small range of distances $(\sim 0.2 \AA)$ above the equilibrium height. This happens in spite of the significantly increased energetic cost to do so, as is evidenced by the calculated planarization energy in Figure $4 \mathrm{~b}$ (blue curve).

3.2. Uncovering the Interplay between Adsorption and Planarization. Given the unusual behavior of the ClB$\mathrm{SubPc} / \mathrm{Cu}(111)$ interface (massive molecular distortion, strong hybridization, and unusual shape of the adsorption potential), we focus next on the role of the vdW forces. Here, we started from the repulsive situation, i.e., using solely the PBE functional (in the absence of long-range vdW interactions). Then, we introduced and gradually increased the long-range van der Waals attraction, in this way pulling the molecule toward the surface. This scaling of the van der Waals forces allowed us to follow the gradual transition from an essentially noninteracting metal-molecule system to a situation characterized by a significant distortion of the adsorbate geometry and a strong hybridization between the molecular and metal states.

Technically, the rescaling is achieved by calculating the energy contribution due to long-range van der Waals interactions, analogous to the original Tkatchenko-Scheffler scheme, ${ }^{54}$ while introducing a scaling prefactor $S$ that varies between 0 and $1 . S$ scales the $C_{6, \mathrm{AB}}$ coefficients that govern the 
pairwise interatomic interactions between two atoms $\mathrm{A}$ and $\mathrm{B}$. This yields

$$
E_{\mathrm{vdW}}[\rho(\mathbf{r})]=-\frac{1}{2} \sum_{\mathrm{AB}} S C_{6, \mathrm{AB}}[\rho(\mathbf{r})] R_{\mathrm{AB}}^{-6} f_{\mathrm{AB}}
$$

Here, $R_{\mathrm{AB}}$ is the interatomic distance and $f_{\mathrm{AB}}$ is a Fermi-type damping function that subdues the correction at short range. Notably, the Tkatchenko-Scheffler (TS) approach, like Grimme's D3 scheme, ${ }^{55}$ the Becke-Johnson scheme, ${ }^{56}$ and the scheme from Sato and Nakai, ${ }^{57}$ aims to include the influence of the atom-pair's local chemical environment into the $C_{6}$ coefficients. In TS, this is achieved by making the $C_{6}$ coefficient dependent on the charge density around the atoms to account for the dependence of vdW interactions on the system's local polarizability. ${ }^{58}$ This makes the TS approach particularly useful in the present case, as in the course of changing $S$ the charge distribution at the interface undergoes pronounced changes (vide infra).

The resulting van der Waals contribution to the adsorption energy $E_{\mathrm{vdW}}$ as a function of $S$ is shown in Figure 5a (black curve). Notably, the curve does not increase smoothly with $S$, in contrast to the evolution of the van der Waals energy for an

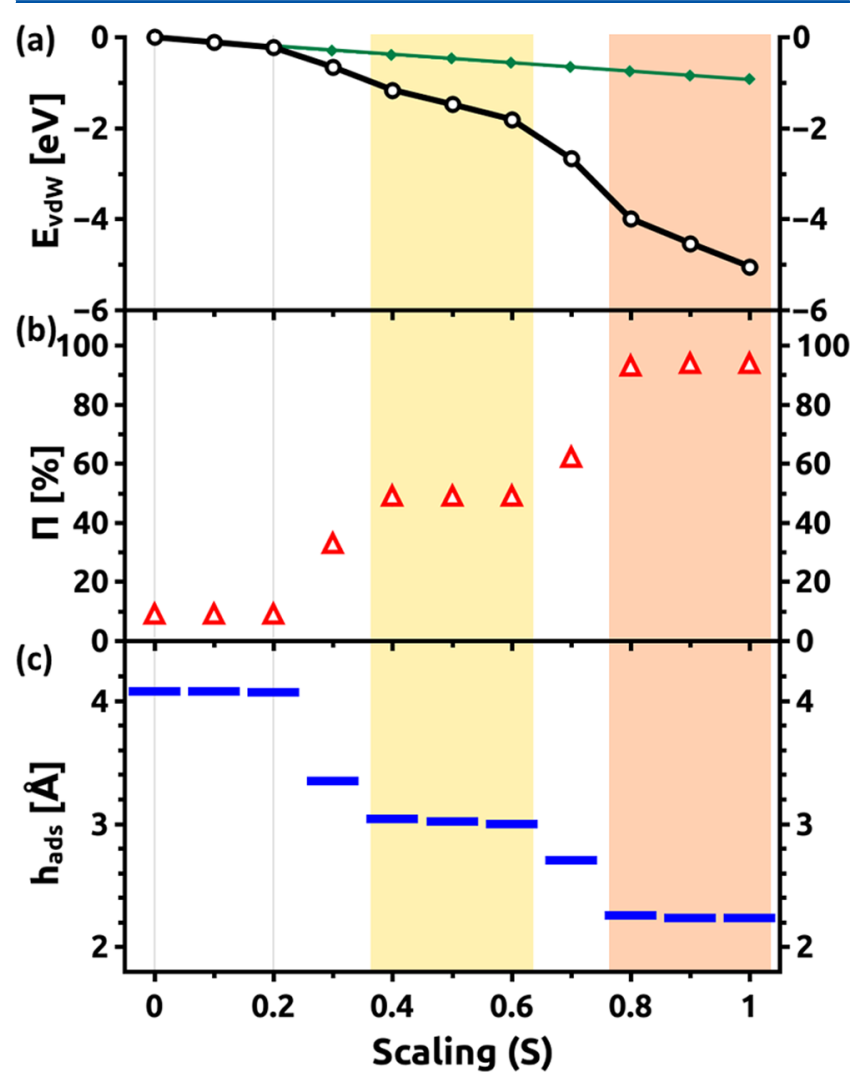

Figure 5. Evolution of the properties of the ClB-SubPc/Cu(111) interface with increasing the $C_{6}$ scaling prefactor $S$. (a) Nonlinear increase in $E_{\mathrm{vdW}}$ (the contribution of the long-range van der Waals interaction to the adsorption energy) as a function of the van der Waals scaling prefactor $S$ (black curve) and linear increase in $E_{\mathrm{vdW}}$ vs $S$ for an isolated molecule in vacuum (green curve). (b) Molecular distortion quantified by the planarity of the adsorbed molecule $(\Pi)$, as defined above. (c) Calculated adsorption height, $h_{\text {ads }}$. There are two distinct regimes with essentially constant planarity and adsorption height ( $S=0.4$ to 0.6 and 0.8 to 1.0 ), shaded in yellow and orange, respectively. isolated molecule (green curve). Rather, there are more pronounced drops between $0.2 \leq S \leq 0.4$ and between $0.6 \leq$ $S \leq 0.8$. These more abrupt changes in energy are accompanied by stepwise increases in the planarity of the ClB-SubPc molecules (Figure 5b) and by stepwise decreases in the adsorption heights (Figure 5c). Consequently, when scaling the $\mathrm{vdW}$ interactions and in this way pulling the molecule toward the surface, one observes two clearly distinct adsorption regimes: Between $S=0.4$ and 0.6, the molecule adsorbs in a somewhat planarized geometry $(\Pi \approx 49 \%)$ at a comparably large distance of around $3.0 \AA$. Conversely, for $S \geq 0.8$, the C18 atoms of ClB-SubPc become essentially planar $(\Pi \approx 94 \%)$ and the adsorption height reduces to $2.2 \AA$. The transition between these regimes coincides with massive modifications of the electronic structure of the interface, as discussed in the following section.

3.3. Interfacial Electronic Structure. A first impression of modifications to the electronic structure of the ClB-SubPc/ $\mathrm{Cu}(111)$ interface caused by increasing the van der Waals attraction can be obtained from the adsorption-induced changes in the work function, $\Delta \Phi$. For the sake of analysis, this quantity can be separated into a contribution from the monolayer due to molecular dipoles, $\Delta \Phi_{\mathrm{ML}}$, and a contribution from the so-called "bond-dipole" resulting from the interaction between substrate and adsorbate, dominated by the adsorptioninduced charge rearrangements, $\Delta \Phi_{\mathrm{BD}} \cdot{ }^{59}$ This yields

$$
\Delta \Phi=\Delta \Phi_{\mathrm{ML}}+\Delta \Phi_{\mathrm{BD}}
$$

In practice, $\Delta \Phi$ and $\Delta \Phi_{\mathrm{ML}}$ are calculated explicitly, and $\Delta \Phi_{\mathrm{BD}}$ is obtained as the difference between these two numbers. Note that the above quantities for the subsystems are calculated in the optimized geometries they adopt upon adsorption. The change in work function for a given system is obtained as the energetic difference between the vacuum levels above and below the calculated slab (for more details see ref 59).

As shown in Figure 6, for small $S, \Delta \Phi$ is positive. This is a consequence of the significant molecular dipole moment

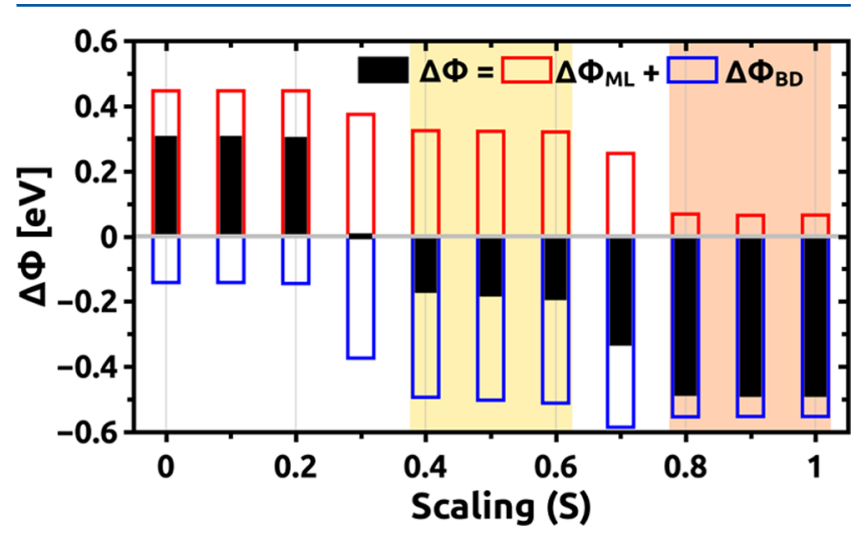

Figure 6. Evolution of the change in work function, $\Delta \Phi$ (black), the contributions due to the molecular layer, $\Delta \Phi_{\mathrm{ML}}$ (red), and the interfacial charge rearrangements, $\Delta \Phi_{\mathrm{BD}}$ (blue), with increasing $C_{6}$ scaling prefactor $S$. The work function of the pristine $\mathrm{Cu}(111)$ surface is calculated to be $4.8 \mathrm{eV}$.

pointing toward the surface, which results in a large positive value of $\Delta \Phi_{\mathrm{ML}}$. As the adsorption distance for small $S$ is large, the change in work function due to the interaction-induced charge rearrangements, $\Delta \Phi_{\mathrm{BD}}$, has only a minor impact. This changes in the first adsorption regime (for $0.4 \leq S \leq 0.6$ ), 
where the absolute value of $\Delta \Phi_{\mathrm{BD}}$ rises by $0.5 \mathrm{eV}$. Simultaneously, the increasing planarity of the adsorbed molecules results in a decreasing molecular dipole and, thus, a smaller $\Delta \Phi_{\mathrm{ML}}$. (The values for the molecular dipoles of the different geometries obtained when varying $S$ can be found in the SI.) As both trends act in the same direction, the overall effect is a change in the sign of the work function modification and a resulting $\Delta \Phi$ of $-0.2 \mathrm{eV}$. This value remains constant over the first adsorption regime.

Upon approaching the second adsorption regime, another step in the work function modification is observed and $\Delta \Phi$ drops to $\sim-0.5 \mathrm{eV}$ (for $0.8 \leq S \leq 1.0$ ). This is primarily a consequence of the additional planarization of the adsorbate molecules resulting in a further reduced molecular dipole (see SI), such that $\Delta \Phi_{\mathrm{ML}}$ becomes $\sim 0.06 \mathrm{eV}$. Notably, the contribution to the work function modification arising from the molecule-metal interaction changes much less, and $\Delta \Phi_{\mathrm{BD}}$ saturates at $-0.55 \mathrm{eV}$. This is quite surprising, considering the massive change in the adsorption distance and the fundamentally different shapes of the interfacial charge rearrangements between the first and second adsorption regimes (vide infra).

A direct comparison of the calculated $\Delta \Phi$ to experiments is difficult, as the experimentally accessible interface contains multiple species, ${ }^{22}$ including dechlorinated molecules (for which we calculate a $\Delta \Phi$ of $-0.8 \mathrm{eV}$ ). Nevertheless, it is interesting to point out that the experimentally determined work function modification at a nominal coverage of 0.5 monolayer equivalents (corresponding to the coverage in the simulations) amounts to $-0.5 \mathrm{eV}^{21}$

The evolution of $\Delta \Phi_{\mathrm{BD}}$ can be understood on the basis of the changes in the energetic alignment between the electronic states in the metal, characterized by the position of the Fermi level, and the states in the adsorbate layer. This is shown in Figure 7.

The trend for $\Delta \Phi_{\mathrm{BD}}$ from Figure 6 is expected to map directly onto the evolution of the level alignment, as demonstrated for a variety of organic molecules on $\operatorname{Ag}(111) .^{60}$ Indeed, up to $S=0.4$, one sees a pronounced shift in the states of the ClB-SubPc monolayer toward higher binding energies (by ca. $0.7 \mathrm{eV}$ ). This can be explained by Pauli pushback $^{61}$ reducing the surface dipole of the metal ${ }^{1}$ and, thus, its work function. Consistent with a constant adsorption height and adsorbate shape, there is no further change in the PDoS up to $S=0.6$. Notably, there is virtually no difference in the shape of the PDoS compared to the effectively noninteracting situation $(S=0)$. Upon further increasing $S$ (i.e., upon pulling the molecule further toward the surface), one again observes a shift of the PDoS toward higher binding energies by $0.4 \mathrm{eV}$. However, like in the above-discussed evolution for $\Delta \Phi_{\mathrm{BD}}$, the net effect is weaker than what is expected based on the strongly decreasing adsorption distance and the resulting massively amplified Pauli pushback. The reason for that is that here one enters the regime of Fermi-level pinning: The formerly unoccupied PDoS of the adsorbate layer is shifted into resonance with the Fermi level, resulting in electron transfer from the metal to the molecules such that the resulting dipole counteracts the effect of Pauli pushback and the overall interfacial dipole remains largely constant. ${ }^{62}$

On the basis of these observations, one can denote the first adsorption regime $(0.4 \leq S \leq 0.6)$ as the "weak-coupling regime" with only negligible charge transfer between molecular states and the metal, as is usually observed for physisorption.

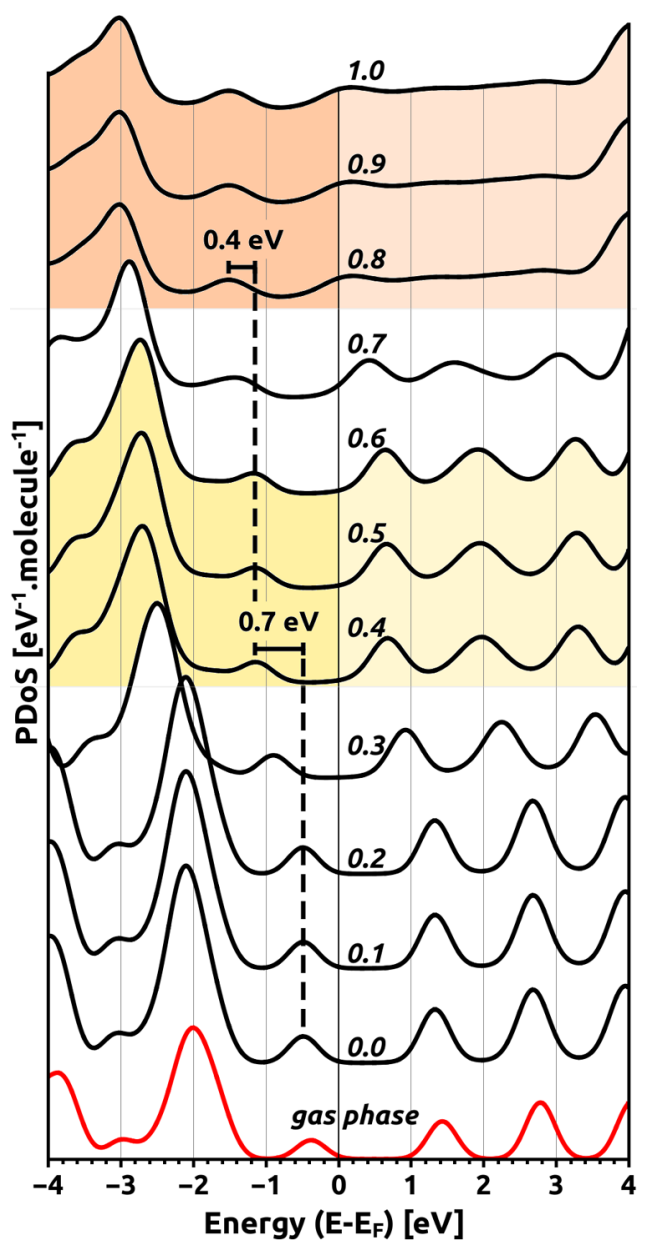

Figure 7. Density of states projected onto the molecular region (PDoS) for the ClB-SubPc/Cu(111) interface as a function of the van der Waals scaling prefactor $S$. The DoS of the gas-phase molecule has been Gaussian broadened and shifted such that the vacuum level of the molecule is the same as that of a clean $\mathrm{Cu}$ slab. The Fermi level of that slab is then used as the energy reference for the isolated molecule in the above plot. This corresponds to assuming vacuum-level alignment and describes the situation for an isolated molecule far away from the $\mathrm{Cu}$ surface, i.e., in the absence of electronic interactions between substrate and adsorbate. The shading denotes the different adsorption regimes (cf., Figures 5 and 6).

Here, it should be stressed that this regime is not merely a curiosity resulting from the scaling of the van der Waals attraction. In fact, it is representative of the situation of ClBSubPc adsorbing on less reactive metals, as is shown in the SI for the ClB-SubPc/ $\mathrm{Ag}(111)$ interface, or on other even more inert substrates like HOPG (see Figure 3).

The second regime $(0.8 \leq S \leq 1.0)$ is then denoted as the "strong-coupling regime", as there we see the partial filling of the molecular PDoS suggesting that electrons have been transferred from the $\mathrm{Cu}$ substrate into previously unoccupied orbitals of the molecular layer. Moreover, a massive broadening of all molecule-derived features in the PDOS occurs. As mentioned earlier, the latter is indicative of a particularly strong hybridization between molecular and metal states. However, we refrain from associating it with chemisorption, as in spite of the significant interaction between the ClB-SubPc molecules and the $\mathrm{Cu}(111)$ substrate, Figure 4a suggests that there would be no bonding in the absence of van der Waals attraction. That is, we face a situation of strong electronic coupling between 
substrate and adsorbate that by itself would, however, not be sufficient to trigger adsorption.

To further distinguish between the two adsorption regimes and to trace the strong hybridization between substrate and adsorbate states, it is useful to consider the adsorption-induced interfacial charge rearrangements, $\Delta \rho$. These are obtained via

$$
\Delta \rho=\rho_{\text {inter }}-\left(\rho_{\text {slab }}+\rho_{\text {mol }}\right)
$$

where $\rho_{\text {inter }}$ is the charge density of the interacting metalmolecule system, $\rho_{\text {slab }}$ is the charge density of only the metal slab, and $\rho_{\text {mol }}$ is the charge density of a molecule in the isolated monolayer (again, all in the geometry obtained for the interacting system). $\Delta \rho$ can be further averaged/integrated along one or two spatial directions to obtain two-dimensional (2D) or one-dimensional (1D) charge rearrangement plots, respectively.

The situation for the weak-coupling regime $(S=0.4)$ is shown in Figure 8a. The charge rearrangements indicate the
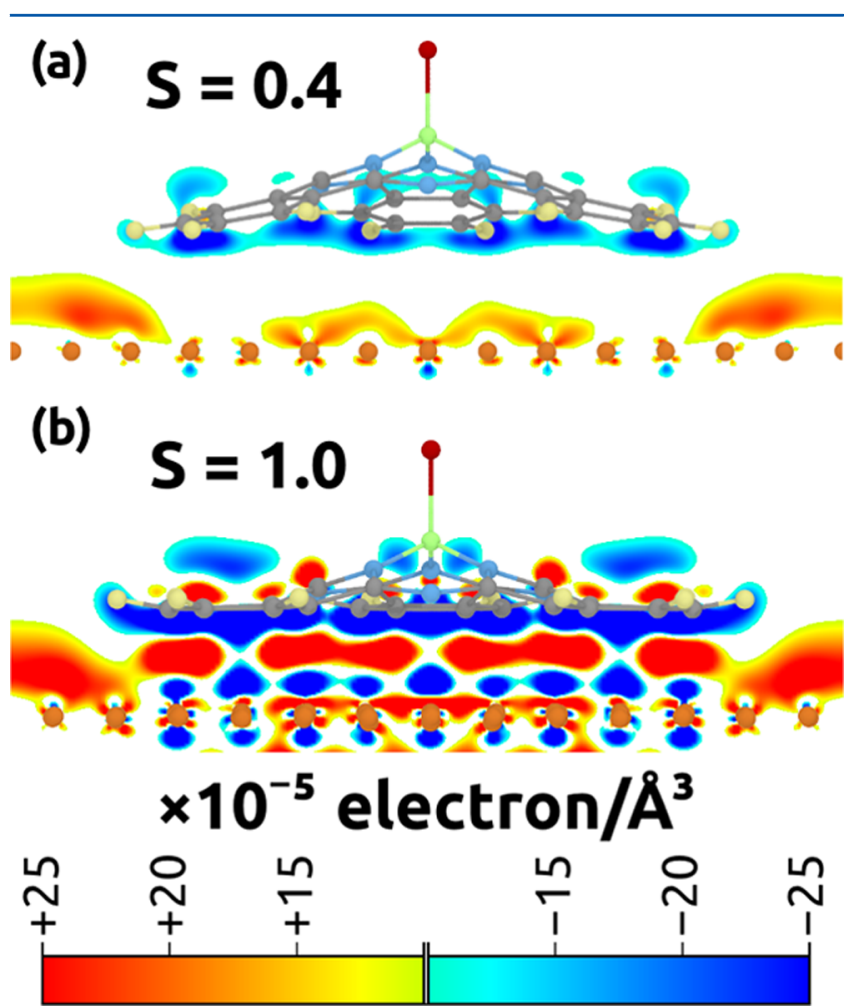

Figure 8. Side view of the interfacial charge rearrangements for (a) $S=$ 0.4 and (b) $S=1.0$ averaged over the unit cell along the direction perpendicular to the plane of projection.

dominance of Pauli pushback with a pronounced electrondensity depletion in the region of the overlap between the tailing electron cloud of the metal and the $\pi$-states of the molecules. The effect is stronger below the plane of the molecule, as there the tailing states from the metal are more prevalent than above. The "pushed-back" electrons are accumulated directly above the metal surface (see also plot of plane-integrated 1D charge rearrangements contained in the SI).

The situation changes qualitatively in the strong-coupling regime, shown for $S=1.0$ in Figure $8 \mathrm{~b}$. There, in addition to the electron-density depletion immediately below the molecular backbone, one observes accumulation directly above the molecule and, even more pronounced, in the region about halfway between the C-18 atoms and the topmost layer of the $\mathrm{Cu}(111)$ substrate. Especially, the latter deserves attention: As shown in Figure 9a, electron accumulation occurs in those
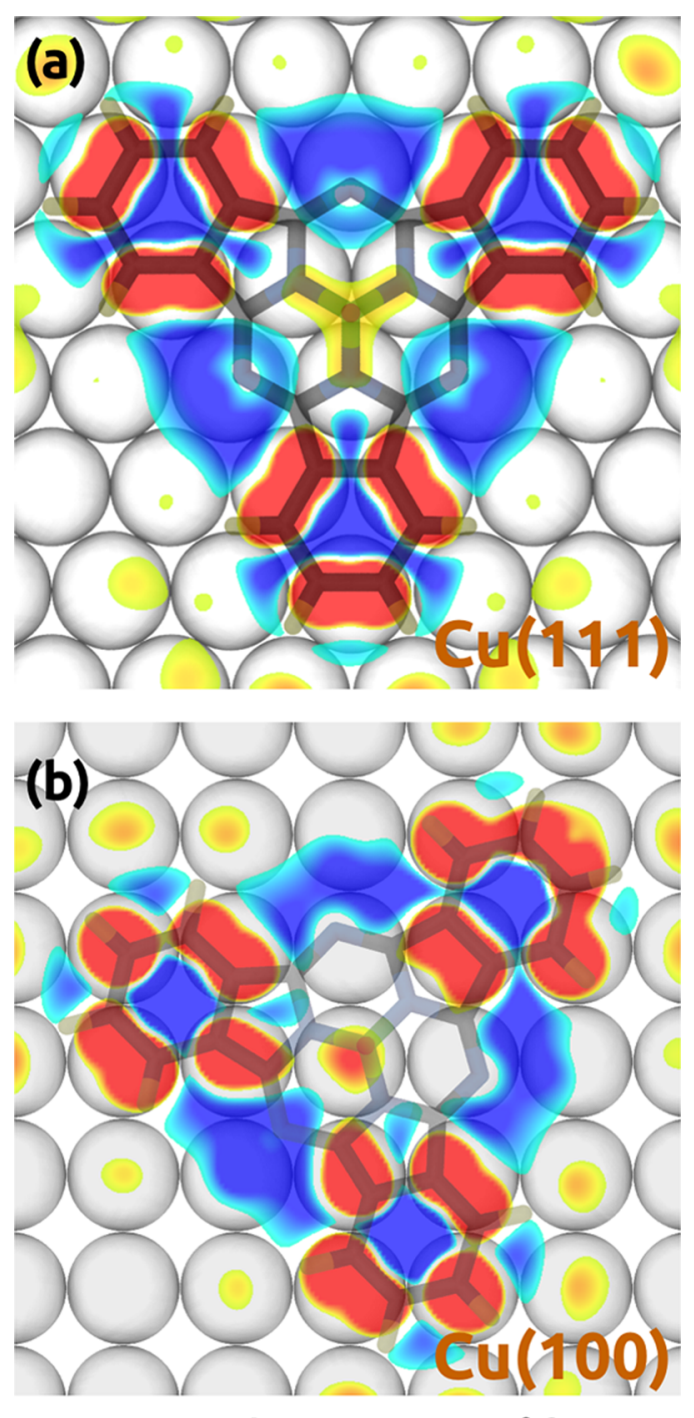

\section{$\times 10^{-4}$ electron $/ \AA^{3}$}

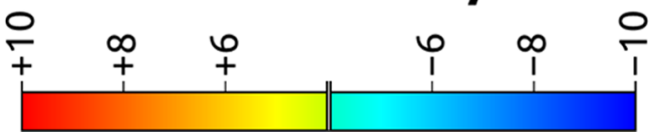

Figure 9. Top view of the charge rearrangements induced by $\mathrm{ClB}$ SubPc on (a) $\mathrm{Cu}(111)$ and (b) $\mathrm{Cu}(100)$. The graphs show a 2D slice of the charge rearrangements calculated for the plane halfway between the molecule and the metal.

regions where the $\mathrm{C}-18$ atoms and the $\mathrm{Cu}$ atoms of the topmost metal layer are particularly close, whereas there is depletion above the bridge and hollow sites of the substrate. The assessment that the driving force for electron accumulation in specific regions is the local proximity between the molecular $\pi$-system and on-top metal sites is confirmed by the very different shape of the charge rearrangement pattern for ClBSubPc on the fourfold symmetric $\mathrm{Cu}(100)$ surface shown in Figure 9b.

We also find that the $\mathrm{H}$ atoms are located $0.2 \AA$ above the C18 plane and that the carbon-carbon bond lengths within the rings constituting the $\mathrm{C}-18$ system increase on average by $\sim 3$ 
$\mathrm{pm}$. These are further indications for a minor disruption of the molecular $\pi$-system caused by the hybridization of the molecular and metal states.

3.4. Role of Molecular Planarity in its Ability to Hybridize. Even though the above results provide evidence that the planarization of the ClB-SubPc molecule is an integral aspect of the adsorption process, the data presented thus far do not fully clarify how crucial planarization actually is for the electronic structure of the interface and especially for the strong hybridization of molecular and metal states. To address this question, we simulated the adsorption of a rigidified ClB-SubPc molecule. For this, we fixed the lateral positions of the carbon atoms in the gas-phase geometry of the molecule, thereby reducing the degrees of freedom during geometry relaxation. In other words, the $\mathrm{C}$ atoms could relax solely along the direction perpendicular to the surface, thereby largely preventing a planarization of the molecule. Care was taken to align the rigidified molecule such that its registry with the $\mathrm{Cu}(111)$ surface was identical to that of the fully relaxed case. The optimum adsorption height for the rigidified molecule rose to 3.0 $\AA$, $0.77 \AA$ more than the fully relaxed structure (vide supra). Interestingly, this adsorption height is almost identical to that obtained for the fully optimized interface in the weak-coupling regime (calculated in the full optimizations for $0.4 \leq S \leq 0.6$ ). Therefore, in Figure 10a, we compare the PDoS of the fully optimized and rigidified structures (both calculated at $S=1.0$ ) with the situation obtained for the weak-coupling case $(S=0.4$, fully optimized).

Intriguingly, we find an almost perfect correspondence between the latter two cases in the PDoSes as well as in the work function modifications $(\Delta \Phi=-0.14 \mathrm{eV}$ for the rigidified molecule and $-0.17 \mathrm{eV}$ for weak-coupling regime). Likewise, the charge rearrangements for the rigidified system show no indication of strong hybridization (Figure 10b). Rather, one solely observes Pauli pushback, as displayed already in Figure 8a for the weak-coupling regime. This clearly shows that the ability of the nonplanar ClB-SubPc to strongly couple to the metal substrate crucially depends on its ability to planarize, which occurs only when van der Waals attractions pull the molecule toward the surface.

\section{SUMMARY AND CONCLUSIONS}

The ClB-SubPc/ $\mathrm{Cu}(111)$ interface provides valuable insight into how changes in an adsorbate geometry induced by the van der Waals attraction to a substrate can change the electronic coupling between substrate and adsorbate, pushing the interface into a significantly different coupling regime. To better understand the peculiarities of this interface, we adopted a strategy that allowed us to scale the van der Waals interactions, to gradually pull the molecule toward the substrate. This revealed two adsorption regimes, which we identified to be cases of weak and strong interfacial coupling. In the former, the originally shuttlecock-shaped molecules are planarized to a lesser degree, and the electronic interaction with the substrate is dominated by Pauli pushback. This, together with the molecular dipoles, determines the adsorbate-induced work function modification. In the second regime, the molecule is pulled very close to the surface, it is largely planarized, and a particularly strong hybridization between molecular and metal states occurs in conjunction with electron transfer from the substrate to adsorbate. In this case, the adsorbate layer becomes metallic, and the work function is determined by Fermi-level pinning. Notably, we show that this regime cannot be
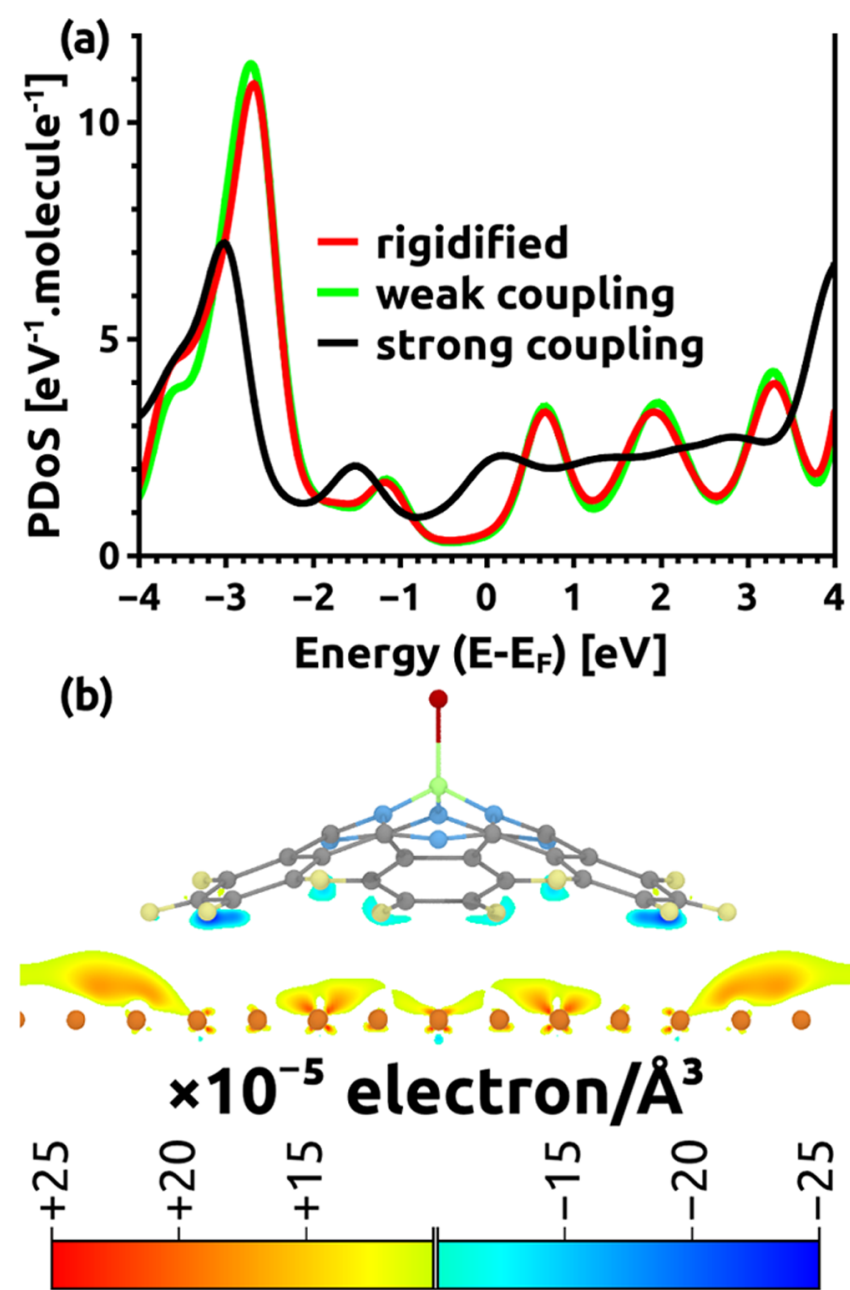

Figure 10. (a) Comparison of the molecule-projected densities of states for the artificially rigidified molecule $(S=1.0)$ and the fully relaxed structures in the weak $(S=0.4)$ - and strong $(S=1.0)$-coupling regimes. (b) Side view of the interfacial charge rearrangements for the rigidified molecule averaged over the unit cell along the direction perpendicular to the plane of projection.

characterized as conventional chemisorption, as in spite of the sizable electronic interactions between substrate and adsorbate, the molecule would not attach to the surface in the absence of van der Waals attraction. In fact, the strongcoupling regime can be reached only provided that the molecule significantly changes its shape, which is shown to be a consequence of van der Waals forces pulling it toward the surface. Thus, the present system can be regarded as an intriguing example of a van der Waals-enabled strong electronic coupling between a metal surface and an organic adsorbate molecule.

\section{ASSOCIATED CONTENT}

\section{Supporting Information}

The Supporting Information is available free of charge on the ACS Publications website at DOI: 10.1021/acs.jpcc.8b03675.

Comparison of chlorinated and dechlorinated systems in terms of quantifiable results, molecular registry with surface, and interfacial electronic structure; algebraic procedure used for calculating $E_{\mathrm{vdW}}$; relationship between the molecule's geometry and its dipole moment and density of states; coordinates of the gas-phase geometry 
of the molecule; table on how van der Waals interactions supply the energy used for planarization; adsorptioninduced charge redistribution in $1 \mathrm{D}$ and $2 \mathrm{D}$; comparison between adsorptions of ClB-SubPc on $\mathrm{Cu}(111)$ and on $\mathrm{Ag}(111)$; and the results for scaling the $C_{6}$ coefficients for a scheme with geometry and charge-density independent interaction coefficients are available (PDF).

\section{AUTHOR INFORMATION}

\section{Corresponding Author}

*E-mail: egbert.zojer@tugraz.at.

\section{ORCID $\odot$}

Shashank S. Harivyasi: 0000-0002-2174-5226

Oliver T. Hofmann: 0000-0002-2120-3259

Oliver L. A. Monti: 0000-0002-0974-7253

Egbert Zojer: 0000-0002-6502-1721

\section{Notes}

The authors declare no competing financial interest.

\section{ACKNOWLEDGMENTS}

The research in this paper was supported by the European Union Seventh Framework Programme Initial Training Network THINFACE under grant agreement number 607232 and by the Austrian Science Fund (FWF): I2081-N20 and P27868N36. O.L.A.M. and N.I. gratefully acknowledge support by the US National Science Foundation under grant numbers CHE1213243 and CHE-1565497. All calculations were carried out using facilities of the Vienna Scientific Cluster. We would also like to thank Elisabeth Wruss for insightful discussions about adsorption of Pcs on coinage metals.

\section{REFERENCES}

(1) Ishii, H.; Sugiyama, K.; Ito, E.; Seki, K. Energy Level Alignment and Interfacial Electronic Structures at Organic/Metal and Organic/ Organic Interfaces. Adv. Mater. 1999, 11, 605-625.

(2) Braun, S.; Salaneck, W. R.; Fahlman, M. Energy-Level Alignment at Organic/Metal and Organic/Organic Interfaces. Adv. Mater. 2009, $21,1450-1472$.

(3) Koch, N. Organic Electronic Devices and Their Functional Interfaces. ChemPhysChem 2007, 8, 1438-1455.

(4) Bagus, P. S.; Hermann, K.; Wöll, C. The interaction of $\mathrm{C}_{6} \mathrm{H}_{6}$ and $\mathrm{C}_{6} \mathrm{H}_{12}$ with noble metal surfaces: Electronic level alignment and the origin of the interface dipole. J. Chem. Phys. 2005, 123, No. 184109.

(5) Toyoda, K.; Nakano, Y.; Hamada, I.; Lee, K.; Yanagisawa, S.; Morikawa, Y. First-principles study of benzene on noble metal surfaces: Adsorption states and vacuum level shifts. Surf. Sci. 2009, 603, 2912-2922.

(6) Liu, W.; Carrasco, J.; Santra, B.; Michaelides, A.; Scheffler, M.; Tkatchenko, A. Benzene adsorbed on metals: Concerted effect of covalency and van der Waals bonding. Phys. Rev. B 2012, 86, No. 245405.

(7) Toyoda, K.; Hamada, I.; Lee, K.; Yanagisawa, S.; Morikawa, Y. Density functional theoretical study of pentacene/noble metal interfaces with van der Waals corrections: Vacuum level shifts and electronic structures. J. Chem. Phys. 2010, 132, No. 134703.

(8) Tautz, F. S. Structure and bonding of large aromatic molecules on noble metal surfaces: The example of PTCDA. Prog. Surf. Sci. 2007, $82,479-520$.

(9) Romaner, L.; Nabok, D.; Puschnig, P.; Zojer, E.; Ambrosch-Draxl, C. Theoretical study of PTCDA adsorbed on the coinage metal surfaces, $\mathrm{Ag}(111), \mathrm{Au}(111)$ and $\mathrm{Cu}(111)$. New J. Phys. 2009, 11, No. 053010.
(10) Monti, O. L. A.; Steele, M. P. Influence of electrostatic fields on molecular electronic structure: insights for interfacial charge transfer. Phys. Chem. Chem. Phys. 2010, 12, 12390-12400.

(11) Yanagisawa, S.; Hamada, I.; Lee, K.; Langreth, D. C.; Morikawa, Y. Adsorption of $\mathrm{Alq}_{3}$ on $\mathrm{Mg}(001)$ surface: Role of chemical bonding, molecular distortion, and van der Waals interaction. Phys. Rev. B 2011, 83, No. 235412.

(12) del Rey, B.; Keller, U.; Torres, T.; Rojo, G.; Agulló-López, F.; Nonell, S.; Martí, C.; Brasselet, S.; Ledoux, I.; Zyss, J. Synthesis and Nonlinear Optical, Photophysical, and Electrochemical Properties of Subphthalocyanines. J. Am. Chem. Soc. 1998, 120, 12808-12817.

(13) Sastre, A.; Torres, T.; Díaz-García, M. A.; Agulló-López, F.; Dhenaut, C.; Brasselet, S.; Ledoux, I.; Zyss, J. Subphthalocyanines: Novel Targets for Remarkable Second-Order Optical Nonlinearities. J. Am. Chem. Soc. 1996, 118, 2746-2747.

(14) Morse, G. E.; Bender, T. P. Boron Subphthalocyanines as Organic Electronic Materials. ACS Appl. Mater. Interfaces 2012, 4, 5055-5068.

(15) El-Khouly, M. E.; Kim, J.-H.; Kim, J.-H.; Kay, K.-Y.; Fukuzumi, S. Subphthalocyanines as Light-Harvesting Electron Donor and Electron Acceptor in Artificial Photosynthetic Systems. J. Phys. Chem. C 2012, 116, 19709-19717.

(16) Schwarze, M.; Tress, W.; Beyer, B.; Gao, F.; Scholz, R.; Poelking, C.; Ortstein, K.; Gunther, A. A.; Kasemann, D.; Andrienko, $\mathrm{D}$; et al. Band structure engineering in organic semiconductors. Science 2016, 352, 1446-1449.

(17) Mannsfeld, S.; Reichhard, H.; Fritz, T. LEED and STM investigation of chloro(subphthalocyaninato)boron on $\mathrm{Au}(111)$. Surf. Sci. 2003, 525, 215-221.

(18) Berner, S.; de Wild, M.; Ramoino, L.; Ivan, S.; Baratoff, A.; Güntherodt, H.-J.; Suzuki, H.; Schlettwein, D.; Jung, T. A. Adsorption and two-dimensional phases of a large polar molecule: Subphthalocyanine on $\operatorname{Ag}(111)$. Phys. Rev. B 2003, 68, No. 115410.

(19) Jiang, N.; Wang, Y.; Liu, Q.; Zhang, Y.; Deng, Z.; Ernst, K.-H.; Gao, H.-J. Polymorphism and chiral expression in two-dimensional subphthalocyanine crystals on $\mathrm{Au}(111)$. Phys. Chem. Chem. Phys. 2010, $12,1318-1322$.

(20) Trelka, M.; Medina, A.; Écija, D.; Urban, C.; Gröning, O.; Fasel, R.; Gallego, J. M.; Claessens, C. G.; Otero, R.; Torres, T.; et al. Subphthalocyanine-based nanocrystals. Chem. Commun. 2011, 47, 9986-9988.

(21) Ilyas, N.; Monti, O. L. A. Interplay of local and global interfacial electronic structure of a strongly coupled dipolar organic semiconductor. Phys. Rev. B 2014, 90, No. 125435.

(22) Ilyas, N.; Harivyasi, S. S.; Zahl, P.; Cortes, R.; Hofmann, O. T.; Sutter, P.; Zojer, E.; Monti, O. L. A. Sticking with the Pointy End? Molecular Configuration of Chloro Boron-Subphthalocyanine on $\mathrm{Cu}(111)$. J. Phys. Chem. C 2016, 120, 7113-7121.

(23) Blum, V.; Gehrke, R.; Hanke, F.; Havu, P.; Havu, V.; Ren, X.; Reuter, K.; Scheffler, M. Ab initio molecular simulations with numeric atom-centered orbitals. Comput. Phys. Commun. 2009, 180, 21752196.

(24) Perdew, J. P.; Burke, K.; Ernzerhof, M. Generalized Gradient Approximation Made Simple. Phys. Rev. Lett. 1996, 77, 3865-3868.

(25) Ruiz, V. G.; Liu, W.; Zojer, E.; Scheffler, M.; Tkatchenko, A. Density-Functional Theory with Screened van der Waals Interactions for the Modeling of Hybrid Inorganic-Organic Systems. Phys. Rev. Lett. 2012, 108, No. 146103.

(26) Schlüter, M.; Chelikowsky, J. R.; Louie, S. G.; Cohen, M. L. Selfconsistent pseudopotential calculations for $\mathrm{Si}$ (111) surfaces: Unreconstructed $(1 \times 1)$ and reconstructed $(2 \times 1)$ model structures. Phys. Rev. B 1975, 12, 4200-4214.

(27) Neugebauer, J.; Scheffler, M. Adsorbate-substrate and adsorbateadsorbate interactions of $\mathrm{Na}$ and $\mathrm{K}$ adlayers on $\mathrm{Al}(111)$. Phys. Rev. $B$ 1992, 46, 16067-16080.

(28) Fu, C.-L.; Ho, K.-M. First-principles calculation of the equilibrium ground-state properties of transition metals: Applications to $\mathrm{Nb}$ and Mo. Phys. Rev. B 1983, 28, 5480-5486. 
(29) Kera, S.; Yamane, H.; Honda, H.; Fukagawa, H.; Okudaira, K. K.; Ueno, N. Photoelectron fine structures of uppermost valence band for well-characterized ClAl-phthalocyanine ultrathin film: UPS and MAES study. Surf. Sci. 2004, 566-568, 571-578.

(30) Fukagawa, H.; Yamane, H.; Kera, S.; Okudaira, K. K.; Ueno, N. UPS fine structures of highest occupied band in vanadylphthalocyanine ultrathin film. J. Electron Spectrosc. Relat. Phenom. 2005, 144-147, 475-477.

(31) Fukagawa, H.; Yamane, H.; Kera, S.; Okudaira, K. K.; Ueno, N. Experimental estimation of the electric dipole moment and polarizability of titanyl phthalocyanine using ultraviolet photoelectron spectroscopy. Phys. Rev. B 2006, 73, No. 041302(R).

(32) Baran, J. D.; Larsson, J. A.; Woolley, R. A. J.; Cong, Y.; Moriarty, P. J.; Cafolla, A. A.; Schulte, K.; Dhanak, V. R. Theoretical and experimental comparison of $\mathrm{SnPc}, \mathrm{PbPc}$, and $\mathrm{CoPc}$ adsorption on Ag(111). Phys. Rev. B 2010, 81, No. 075413.

(33) Cai, Y.; Qiao, X. Adsorption and reconstruction of metalphthalocyanine molecules on $\mathrm{Pt}(001)$ investigated by density functional theory. Surf. Sci. 2014, 630, 202-207.

(34) Cuadrado, R.; Cerdá, J. I.; Wang, Y.; Xin, G.; Berndt, R.; Tang, $\mathrm{H}$. CoPc adsorption on $\mathrm{Cu}(111)$ : Origin of the $\mathrm{C} 4$ to $\mathrm{C} 2$ symmetry reduction. J. Chem. Phys. 2010, 133, No. 154701.

(35) Annese, E.; Fujii, J.; Vobornik, I.; Rossi, G. Structure and Electron States of Co-phthalocyanine Interacting With the $\mathrm{Cu}(111)$ Surface. J. Phys. Chem. C 2011, 115, 17409-17416.

(36) Baran, J. D.; Larsson, J. A. Structure and Energetics of Shuttlecock-Shaped Tin-Phthalocyanine on $\operatorname{Ag}(111)$ : A Density Functional Study Employing Dispersion Correction. J. Phys. Chem. C 2012, 116, 9487-9497.

(37) Niu, T.; Zhou, M.; Zhang, J.; Feng, Y.; Chen, W. Dipole Orientation Dependent Symmetry Reduction of Chloroaluminum Phthalocyanine on $\mathrm{Cu}(111)$. J. Phys. Chem. C 2013, 117, 1013-1019.

(38) Wruss, E.; Hofmann, O. T.; Egger, D. A.; Verwüster, E.; Gerlach, A.; Schreiber, F.; Zojer, E. Adsorption Behavior of Nonplanar Phthalocyanines: Competition of Different Adsorption Conformations. J. Phys. Chem. C 2016, 120, 6869-6875.

(39) Shen, K.; Narsu, B.; Ji, G.; Sun, H.; Hu, J.; Liang, Z.; Gao, X.; Li, H.; Li, Z.; Song, B.; et al. On-surface manipulation of atom substitution between cobalt phthalocyanine and the $\mathrm{Cu}(111)$ substrate. RSC Adv. 2017, 7, 13827-13835.

(40) Maughan, B.; Zahl, P.; Sutter, P.; Monti, O. L. A. Configurationspecific electronic structure of strongly interacting interfaces: TiOPc on $\mathrm{Cu}(110)$. Phys. Rev. B 2017, 96, No. 235133.

(41) Motai, K.; Hashizume, T.; Shinohara, H.; Saito, Y.; Pickering, H. W.; Nishina, Y.; Sakurai, T. $\mathrm{C}_{60}$ Grown on the $\mathrm{Cu}(111) 1 \times 1$ Surface. Jpn. J. Appl. Phys. 1993, 32, L450-L453.

(42) Zoppi, L.; Garcia, A.; Baldridge, K. K. Theoretical Investigation of the Binding Process of Corannulene on a $\mathrm{Cu}(111)$ Surface $\dagger$. J. Phys. Chem. A 2010, 114, 8864-8872.

(43) Shi, X.-Q.; Li, Y.; Van Hove, M. A.; Zhang, R.-Q. Interactions between Organics and Metal Surfaces in the Intermediate Regime between Physisorption and Chemisorption. J. Phys. Chem. C 2012, 116, 23603-23607.

(44) Ferretti, A.; Baldacchini, C.; Calzolari, A.; Di Felice, R.; Ruini, A.; Molinari, E.; Betti, M. G. Mixing of Electronic States in Pentacene Adsorption on Copper. Phys. Rev. Lett. 2007, 99, No. 046802.

(45) Ules, T.; Lüftner, D.; Reinisch, E. M.; Koller, G.; Puschnig, P.; Ramsey, M. G. Orbital tomography of hybridized and dispersing molecular overlayers. Phys. Rev. B 2014, 90, No. 155430.

(46) Müller, K.; Seitsonen, A. P.; Brugger, T.; Westover, J.; Greber, T.; Jung, T.; Kara, A. Electronic Structure of an Organic/Metal Interface: Pentacene/ $\mathrm{Cu}(110)$. J. Phys. Chem. C 2012, 116, 2346523471.

(47) Duhm, S.; Gerlach, A.; Salzmann, I.; Bröker, B.; Johnson, R. L.; Schreiber, F.; Koch, N. PTCDA on $\mathrm{Au}(111), \mathrm{Ag}(111)$ and $\mathrm{Cu}(111)$ : Correlation of interface charge transfer to bonding distance. Org. Electron. 2008, 9, 111-118.
(48) Lorente, N.; Hedouin, M. F. G.; Palmer, R. E.; Persson, M. Chemisorption of benzene and STM dehydrogenation products on $\mathrm{Cu}(100)$. Phys. Rev. B 2003, 68, No. 155401.

(49) Ugolotti, A.; Harivyasi, S. S.; Baby, A.; Dominguez, M.; Pinardi, A. L.; López, M. F.; Martín-Gago, J. Á; Fratesi, G.; Floreano, L.; Brivio, G. P. Chemisorption of Pentacene on $\mathrm{Pt}(111)$ with a Little Molecular Distortion. J. Phys. Chem. C 2017, 121, 22797-22805.

(50) Baby, A.; Fratesi, G.; Vaidya, S. R.; Patera, L. L.; Africh, C.; Floreano, L.; Brivio, G. P. Anchoring and Bending of Pentacene on Aluminum (001). J. Phys. Chem. C 2015, 119, 3624-3633.

(51) Endres, J.; Pelczer, I.; Rand, B. P.; Kahn, A. Determination of Energy Level Alignment within an Energy Cascade Organic Solar Cell. Chem. Mater. 2016, 28, 794-801.

(52) Koch, N.; Gerlach, A.; Duhm, S.; Glowatzki, H.; Heimel, G.; Vollmer, A.; Sakamoto, Y.; Suzuki, T.; Zegenhagen, J.; Rabe, J. P.; et al. Adsorption-Induced Intramolecular Dipole: Correlating Molecular Conformation and Interface Electronic Structure. J. Am. Chem. Soc. 2008, 130, 7300-7304.

(53) Carrasco, J.; Liu, W.; Michaelides, A.; Tkatchenko, A. Insight into the description of van der Waals forces for benzene adsorption on transition metal (111) surfaces. J. Chem. Phys. 2014, 140, No. 084704.

(54) Tkatchenko, A.; Scheffler, M. Accurate Molecular Van Der Waals Interactions from Ground-State Electron Density and FreeAtom Reference Data. Phys. Rev. Lett. 2009, 102, No. 073005.

(55) Grimme, S.; Antony, J.; Ehrlich, S.; Krieg, H. A consistent and accurate $\mathrm{ab}$ initio parametrization of density functional dispersion correction (DFT-D) for the 94 elements H-Pu. J. Chem. Phys. 2010, 132, No. 154104

(56) Becke, A. D.; Johnson, E. R. A density-functional model of the dispersion interaction. J. Chem. Phys. 2005, 123, No. 154101.

(57) Sato, T.; Nakai, H. Density functional method including weak interactions: Dispersion coefficients based on the local response approximation. J. Chem. Phys. 2009, 131, No. 224104.

(58) Casimir, H. B. G.; Polder, D. The Influence of Retardation on the London-van der Waals Forces. Phys. Rev. 1948, 73, 360-372.

(59) Heimel, G.; Romaner, L.; Zojer, E.; Bredas, J.-L. The Interface Energetics of Self-Assembled Monolayers on Metals. Acc. Chem. Res. 2008, 41, 721-729.

(60) Hofmann, O. T.; Atalla, V.; Moll, N.; Rinke, P.; Scheffler, M. Interface dipoles of organic molecules on $\mathrm{Ag}(111)$ in hybrid densityfunctional theory. New J. Phys. 2013, 15, No. 123028.

(61) Bagus, P. S.; Staemmler, V.; Wöll, C. Exchangelike Effects for Closed-Shell Adsorbates: Interface Dipole and Work Function. Phys. Rev. Lett. 2002, 89, No. 096104.

(62) Hofmann, O. T.; Egger, D. A.; Zojer, E. Work-Function Modification beyond Pinning: When Do Molecular Dipoles Count? Nano Lett. 2010, 10, 4369-4374. 